\title{
A Note on Solid Partitions
}

\author{
By Donald E. Knuth
}

Abstract. The problem of enumerating partitions which satisfy a given partial order relation is reduced to the problem of enumerating permutations satisfying that relation. This theorem is applied to the enumeration of solid partitions; existing tables of solid partitions are extended.

A plane partition of $n$ is an arrangement of nonnegative integers

$$
\begin{array}{cccc}
n_{00} & n_{01} & n_{02} & \cdots \\
n_{10} & n_{11} & n_{12} & \cdots \\
\cdots & \cdots & \cdots & \cdots
\end{array}
$$

which sum to $n$, and which are nonincreasing in both rows and columns:

For example,

$$
n_{i j} \geqq n_{i(i+1)}, \quad n_{i j} \geqq n_{(i+1) i} .
$$

$$
\begin{array}{lllll}
5 & 4 & 2 & 1 & 1 \\
3 & 2 & & & \\
2 & 2 & & &
\end{array}
$$

is a plane partition of 22. (Blank entries are zero.)

Let $b(n)$ denote the total number of plane partitions of $n$. In 1912, Major Percy A. MacMahon triumphantly announced [5] a proof of the remarkable formula

$$
1+b(1) z+b(2) z^{2}+b(3) z^{3}+\cdots=1 /(1-z)\left(1-z^{2}\right)^{2}\left(1-z^{3}\right)^{3} \cdots
$$

which he had previously verified by numerous empirical calculations, but which he had been unable to prove six months earlier [4]. In his enthusiasm he concluded his paper by saying, "We have evidently, potentially, the complete solution of the problem of three-dimensional partition, and it remains to work it out and bring it to the same completeness as has been secured in this Part for the problem in two dimensions. This will form the subject of Part VII of this Memoir." The problem of enumerating three-dimensional ("solid") partitions has never been resolved, however, and Part VII of MacMahon's classic Memoir never appeared. No constructive proof of MacMahon's formula for the two-dimensional case was known until 1969 [2].

MacMahon at one time conjectured that, if $c(n)$ is the number of solid partitions of $n$, the formula

$$
1+c(1) z+c(2) z^{2}+c(3) z^{3}+\cdots=1 /(1-z)\left(1-z^{2}\right)^{3}\left(1-z^{3}\right)^{6}\left(1-z^{4}\right)^{10} \ldots
$$

Received November 5, 1969, revised February 18, 1970.

AMS 1969 subject classifications. Primary 1048, 0510; Secondary 0620.

Key words and phrases. Plane partitions, solid partitions, partially-ordered partitions, partiallyordered permutations, index of permutation, backtracking. 
TABLE 1

\begin{tabular}{rrrrrrr}
\hline$n$ & \multicolumn{1}{c}{$c(n)$} & \multicolumn{1}{c}{$c^{\prime}(n)$} & \multicolumn{1}{c}{$d(n)$} & \multicolumn{1}{c}{$d^{\prime}(n)$} & $e(n)$ & $e^{\prime}(n)$ \\
\hline 0 & 1 & 1 & 1 & 1 & 0 & 0 \\
1 & 1 & 1 & 0 & 0 & 1 & 1 \\
2 & 4 & 4 & 2 & 2 & 3 & 3 \\
3 & 10 & 9 & 5 & 4 & 6 & 5 \\
4 & 26 & 22 & 12 & 9 & 10 & 7 \\
5 & 59 & 46 & 24 & 16 & 15 & 9 \\
6 & 140 & 102 & 56 & 35 & 20 & 10 \\
7 & 307 & 206 & 113 & 63 & 26 & 12 \\
8 & 684 & 427 & 248 & 129 & 34 & 16 \\
9 & 1464 & 841 & 503 & 234 & 46 & 21 \\
10 & 3122 & 1658 & 1043 & 445 & 68 & 29 \\
11 & 6500 & 3173 & 2080 & 798 & 97 & 32 \\
12 & 13426 & 6038 & 4169 & 1458 & 120 & 22 \\
13 & 27248 & 11251 & 8145 & 2568 & 112 & -2 \\
14 & 54804 & 20807 & 15897 & 4561 & 23 & -39 \\
15 & 108802 & 37907 & 30545 & 7924 & -186 & -67 \\
16 & 214071 & 68493 & 58402 & 13770 & -496 & -48 \\
17 & 416849 & 122338 & 110461 & 23584 & -735 & 64 \\
18 & 805124 & 216819 & 207802 & 40301 & -531 & 277 \\
19 & 1541637 & 380637 & 387561 & 68097 & 779 & 576 \\
20 & 2930329 & 663417 & 718875 & 114646 & 3894 & 848 \\
21 & 5528733 & 1147033 & 1324038 & 191336 & 9323 & 981 \\
22 & 10362312 & 1969961 & 2425473 & 317893 & 16472 & 771 \\
23 & 19295226 & 3359677 & 4416193 & 524396 & 23056 & 40 \\
24 & 35713454 & 5694592 & 7999516 & 861054 & 23850 & -1498 \\
25 & 65715094 & 9592063 & 14411507 & 1405130 & 10116 & -4276 \\
26 & 120256653 & 16065593 & 25837198 & 2282651 & -31613 & -8745 \\
27 & 218893580 & 26756430 & 46092306 & 3688254 & -120720 & -15062 \\
28 & 396418699 & 44328414 & 81851250 & 5933463 & -283202 & -21702 \\
\hline & & & & & &
\end{tabular}

might be valid. (See [6, pp. 175-176].) But recently-computed tables [1] show that this formula gives the wrong answer for $n=6$. In order to find out the true nature of $c(n)$, the only known approach is to prepare tables, by brute force, and to examine these tables with the hope of finding some pattern.

The purpose of this note is to describe a slightly sophisticated computational method for extending the existing tables of $c(n)$, by showing how the coefficients $d(n)$ in the formula

$$
\begin{aligned}
& 1+c(1) x+c(2) x^{2}+\cdots \\
& =\left(1+d(1) x+d(2) x^{2}+\cdots\right) /(1-x)\left(1-x^{2}\right)\left(1-x^{3}\right) \cdots
\end{aligned}
$$

can be computed for small $n$. The computational technique we will discuss was essentially used by MacMahon [4] in his examination of the two-dimensional case, 
and later also in his preliminary study of solid partitions [5, pp. 360-373]. Since the ideas apply under fairly general circumstances, we will reformulate MacMahon's method for the case of arbitrary partially-ordered sets.

Theory. Let $P$ be a set of elements that are partially-ordered by the relation $\prec$ and well-ordered by the total order relation $<$. We will assume that the partial order is embedded in the total order, in the sense that

$$
x \prec y \text { implies } x<y \text {. }
$$

By a labelling of $P$ we mean a function $n(x)$ taking the elements of $P$ into the set $N$ of nonnegative integers, satisfying the two conditions

(L1) $x \prec y$ implies $n(x) \geqq n(y)$.

(L2) Only finitely many $x$ have $n(x)>0$.

We wish to count the number of labellings of $P$ satisfying certain restrictions.

It is not difficult to show that there is a one-to-one correspondence between labellings of $P$ and pairs of sequences

$$
\begin{aligned}
& n_{1} \geqq n_{2} \geqq \cdots \geqq n_{m}, \\
& x_{1}, x_{2}, \cdots, x_{m},
\end{aligned}
$$

where $m \geqq 0$, the $n_{i}$ are positive integers, and the $x_{i}$ are distinct elements of $P$, subject to the following two conditions:

(S1) For $1 \leqq j \leqq m$ and $x \in P, x \prec x_{j}$ implies $x=x_{i}$ for some $i<j$.

(S2) $x_{i}>x_{i+1}$ implies $n_{i}>n_{i+1}$, for $1 \leqq i<m$.

To construct such a correspondence, we may proceed as follows. Given a labelling, let $n_{1}, n_{2}, \cdots, n_{m}$ be the nonzero labels in nonincreasing order, and let $x_{i}$ be such that $n\left(x_{i}\right)=n_{i}$; the $x$ 's are arranged so that we put $x$ before $y$ when $n(x)=n(y)$ and $x<y$. Then (S1) is satisfied, since $x \prec x_{i}$ implies that $n(x) \geqq n\left(x_{j}\right)$ and $x<x_{i}$; and (S2) is satisfied, since $n_{i}=n_{i+1}$ implies that $x_{i}<x_{i+1}$. Conversely, given sequences (3) satisfying (S1) and (S2) we define a labelling by setting $n\left(x_{i}\right)=n_{i}$ for $1 \leqq i \leqq m$ and $n(x)=0$ for all other $x$. Clearly (L2) is satisfied, and so is (L1), for if $x \prec y$ we have either $n(x) \geqq 0=n(y)$ or $y=x_{i}, x=x_{i}, i<j, n(x)=n_{i} \geqq n_{i}=n(y)$. It is easy to verify that these two constructions are inverses of each other, since (S2) and the relation $n\left(x_{i}\right)=n_{i}$ uniquely defines the sequence of $x$ 's.

For example, let $P$ be the set of points $\{(i, j) \mid i, j \in N\}$ of the plane, subject to the partial order

$$
(i, j) \preceq\left(i^{\prime}, j^{\prime}\right), \quad \text { if and only if } i \leqq i^{\prime} \text { and } j \leqq j^{\prime},
$$

and the well-order

$$
(i, j)<\left(i^{\prime}, j^{\prime}\right) \text {, if and only if } i<i^{\prime} \text { or }\left(i=i^{\prime} \text { and } j<j^{\prime}\right) .
$$

A labelling of this set $P$ is essentially a plane partition; for example, $(1)$ has $n((0,0))=$ $5, n((0,1))=4, \cdots, n((0,5))=0$, etc. The sequences (3) corresponding to (1) are

$$
\begin{aligned}
& n_{1}, \cdots, n_{m}=5,4,3,2,2,2,2,1,1 ; \\
& x_{1}, \cdots, x_{m}=(0,0),(0,1),(1,0),(0,2),(1,1),(2,0),(2,1),(0,3),(0,4) .
\end{aligned}
$$

We are interested primarily in cases where $P$ is countably infinite, and when 
the labels sum to a given number $n$ :

$$
\sum_{x \in P} n(x)=n \text {. }
$$

Let us say that such a labelling is a P-partition of $n$. For this case we can refine the above correspondence in order to minimize the dependence of the $n$ 's on the $x$ 's. Every $P$-partition of $n$ corresponds uniquely to a pair of sequences

$$
\begin{aligned}
& n_{1} \geqq n_{2} \geqq n_{3} \geqq \cdots \geqq 0, \quad \sum n_{i}=n ; \\
& x_{1}, x_{2}, \cdots, x_{m} ;
\end{aligned}
$$

where $m \geqq 0$, the $n_{i}$ form an infinite sequence of nonnegative integers, the $x_{i}$ are distinct elements of $P$, and conditions (S1), (S2), (S3), and (S4) hold, where

(S3) Either $m=0$ or $n_{m}>n_{m+1}$;

(S4) If $m>0$, there exists $x \in P$ such that $x<x_{m}$ and $x \neq x_{i}$ for $1 \leqq i \leqq m$.

Such pairs of sequences correspond uniquely to pairs of sequences (3) of the former type, when $P$ is infinite, if we extend the $n$ 's by adding infinitely many zeroes, and if we contract the $x$ 's, if necessary, by removing $x_{m}$ if it is the least element of $P-\left\{x_{1}, \cdots, x_{m-1}\right\}$. For example, (4) corresponds to

$$
\begin{aligned}
& 5,4,3,2,2,2,2,1,1,0,0,0, \cdots ; \\
& (0,0),(0,1),(1,0),(0,2),(1,1),(2,0),(2,1) .
\end{aligned}
$$

It is not difficult to verify that this process defines a one-to-one correspondence.

Let us say that a sequence of $x$ 's satisfying conditions (S1) and (S4) is a topological sequence, since such sequences arise in connection with "topological sorting" (see [3, pp. 258-268]). The index of a topological sequence is defined to be

$$
\sum\left\{j \mid 1 \leqq j<m \text { and } x_{i}>x_{i+1}\right\}+m \text {. }
$$

Given a topological sequence, every sequence $n_{1} \geqq n_{2} \geqq \cdots$ of nonnegative integers satisfying (S2) and (S3) corresponds uniquely to a sequence $p_{1} \geqq p_{2} \geqq \cdots$ obtained by subtracting unity from $n_{1}, \cdots, n_{i}$, in turn, for each $j$ such that $x_{i}>x_{i+1}$ or $j=m$.

For example, the topological sequence

$$
x_{1}, \cdots, x_{7}=(0,0),(0,1),(1,0),(0,2),(1,1),(2,0),(2,1)
$$

has index $3+7$; and the sequence $p_{1}, p_{2}, p_{3}$; corresponding to (7) is obtained by subtracting 1 from $n_{1}, n_{2}, n_{3}$, then subtracting 1 from each of $n_{1}, n_{2}, \cdots, n_{7}$ :

$$
p_{1}, p_{2}, p_{3}=3,2,1,1,1,1,1,1,1,0,0,0, \cdots .
$$

Note that the sum of the $p_{i}$ is the sum of the $n_{i}$ diminished by the index; this is the only interdependence between the $x$ 's, the $p$ 's, and $n$.

In summary, our observations have the following consequence:

THEOREM. Let $P$ be an infinite partially-ordered set. There is a one-to-one correspondence between $P$-partitions of $n$, and ordered pairs $(\mathrm{x}, \mathrm{p})$ where $\mathrm{x}=x_{1}, \cdots, x_{\mathrm{m}}$ is a topological sequence and $\mathrm{p}=p_{1}, p_{2}, \cdots$ is a partition (in the ordinary sense) of $n-k, k$ the index of $\mathbf{x}$.

Since the generating function for ordinary partitions is $1 /(1-z)\left(1-z^{2}\right) \cdots$, we have 
COROLlary. Let $P$ be an infinite partially-ordered set; let $s(n)$ be the number of $P$-partitions of $n$, and let $t(k)$ be the number of topological sequences of $P$ having index $k$. Then,

$$
\begin{aligned}
1+s(1) z & +s(2) z^{2}+\cdots \\
& =\left(1+t(1) z+t(2) z^{2}+\cdots\right) /(1-z)\left(1-z^{2}\right)\left(1-z^{3}\right) \cdots .
\end{aligned}
$$

Consequently, we can enumerate $P$-partitions by enumerating only the topological sequences, and the latter are easier to enumerate. Note that the definition of topological sequence involves an assumed well ordering $<$; but the corollary shows that the number of topological sequences of given index is independent of the well ordering. Therefore we can choose any convenient well-ordering relation (e.g., the lexicographic order in our examples) when doing the enumeration.

An Application. To find the number $c(n)$ of solid partitions of $n$, let $P$ be the set of three-dimensional lattice points

$$
\{(i, j, k) \mid i, j, k \in N\},
$$

with the partial ordering

$$
(i, j, k) \preceq\left(i^{\prime}, j^{\prime}, k^{\prime}\right),
$$

if and only if

$$
i \leqq i^{\prime}, \quad j \leqq j^{\prime}, \quad k \leqq k^{\prime} ;
$$

and with the lexicographic well-ordering

$$
(i, j, k)<\left(i^{\prime}, j^{\prime}, k^{\prime}\right) \text {, }
$$

if and only if

$$
i<i^{\prime} \text { or }\left(i=i^{\prime} \text { and } j<j^{\prime}\right) \text { or }\left(i=i^{\prime}, j=j^{\prime} \text {, and } k<k^{\prime}\right) \text {. }
$$

If $d(n)$ is the corresponding number of topological sequences having index $n$, we have the formula

$$
\begin{aligned}
1+c(1) z & +c(2) z^{2}+\cdots \\
& =\left(1+d(1) z+d(2) z^{2}+\cdots\right) /(1-z)\left(1-z^{2}\right)\left(1-z^{3}\right) \cdots .
\end{aligned}
$$

The following ALGOL program enumerates $d(0), d(1), \cdots, d(n)$, using a fairly standard backtracking method:

procedure count $(n, d)$; integer $n$; integer array $d$;

begin comment $d[i]$ is set to the number of topological sequences of index $i$, for $0 \leqq i \leqq n$;

integer array $c c[0: n, 0: n] ; \quad$ comment number of columns in given plane, row; integer array $r r[0: n]$; comment number of rows in given plane;

integer $p p$;

integer $k$; comment number of planes;

integer array row $[0: n]$; comment recursion depth; integer array plane $[0: n]$ comment row of given move; comment plane of given move; 


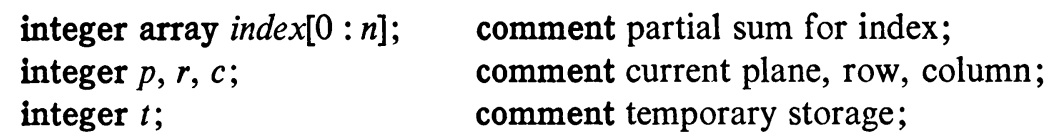
comment temporary storage;

\section{for $p:=0$ step 1 until $n$ do}

begin $r r[p]:=0$; for $r:=0$ step $i$ until $n$ do $c c[p, r]:=0$ end;

for $k:=1$ step 1 until $n$ do $d[k]:=0$;

$p p:=k:=$ index $[0]:=$ plane $[0]:=\operatorname{row}[0]:=0 ; d[0]:=1$;

up $: k:=k+1 ; p:=p p ; r:=0$;

try $: c:=c c[p, r]$

if $p>0$ then if $c c[p-1, r] \leqq c$ then go to again;

if $r>0$ then if $c c[p, r-1] \leq c$ then go to again;

if $p<$ plane $[k-1]$ then go to less;

if $p=$ plane[ $k-1]$ then if $r<\operatorname{row}[k-1]$ then go to less;

$t:=$ index $[k-1]$; go to move;

less: $t:=$ index $[k-1]+k-1$;

if $k+t>n$ then go to nope;

move: begin comment We have now decided to choose the point $(p, r, c)$ as

the $k$ th element of the topological sequence; end;

index $[k]:=t$;

if $p+r>0$ then $d[k+t]:=d[k+t]+1$;

if $t+k \geqq n$ then go to again;

if $r+c=0$ then $p p:=p p+1$;

if $c=0$ then $\operatorname{rr}[p]:=\operatorname{rr}[p]+1$;

$c c[p, r]:=c+1$

plane $[k]:=p ;$ row $[k]:=r$; go to $u p$;

again: if $r>0$ then begin $r:=r-1$; go to try end;

if $p>0$ then begin $p:=p-1 ; r:=\operatorname{rr}[p]$; go to try end;

nope: $k:=k-1$;

if $k>0$ then begin $p:=$ plane $k] ; r:=\operatorname{row}[k]$;

$$
c:=c c[p, r]-1 ; c c[p, r]:=c ;
$$

if $c=0$ then $\operatorname{rr}[p]:=\operatorname{rr}[p]-1$;

if $r+c=0$ then $p p:=p p-1$;

go to again end;

end count.

Simple modifications to this program make it possible to count $c^{\prime}(n)$, the number of solid partitions restricted to at most two planes. Table 1 shows the values of $c(n)$, $c^{\prime}(n), d(n), d^{\prime}(n), e(n)$ and $e^{\prime}(n)$, for $n \leqq 28$, where the exponents $e(n)$ are defined by the relation

$$
1+c(1) z+c(2) z^{2}+\cdots=1 /(1-z)^{\circ(1)}\left(1-z^{2}\right)^{\bullet(2)}\left(1-z^{3}\right)^{\circ(3)} \cdots .
$$

The numbers $d^{\prime}(n), e^{\prime}(n)$ are defined similarly. Unfortunately no pattern is evident in these numbers, so this table should suffice to disprove most simple conjectures about solid partitions. 
1. A. O. L. Atkin, P. Bratley, I. G. MacDonald \& J. K. S. McKay, "Some computations for $m$-dimensional partitions," Proc. Cambridge Philos. Soc., v. 63, 1967, pp. 1097-1100. MR 36 \#124.

2. Edward A. Bender \& Donald E. KNUTH, "Enumeration of plane partitions," J. Combinatorial Theory. (To appear.)

3. Donald E. KNUth, The Art of Computer Programming. Vol. 1, Addison-Wesley, Reading, Mass., 1968.

4. MAJOR P. A. MACMAHON, "Memoir on the theory of the partitions of numbers. V: Partitions in two-dimensional space," Philos. Trans. Roy. Soc. London Ser. A, v. 211, 1912, pp. 75-110.

5. MAJOR P. A. MACMAHON, "Memoir on the theory of the partitions of numbers. VI: Partitions in two-dimensional space, to which is added an adumbration of the theory of the partitions in three-dimensional space," Philos. Trans. Roy. Soc. London Ser. A, v. 211, 1912, pp. 345-373.

6. Major P. A. MacMahon, Combinatory Analysis. Vol. 2, Cambridge Univ. Press, Cambridge, 1916; reprint, Chelsea, New York, 1960. 